\title{
The Regulatory Effect of ERK1 Pathway in the DNA Hypomethylation of MRL/lpr Mice
}

\author{
Miaoxuan Luo ${ }^{1, \dagger}$, Shanshan Wei ${ }^{2,}$, Xiangbin $\mathrm{Mi}^{2,}$, , Tangde Zhang ${ }^{2}$, Wu Zhang ${ }^{1}$ \\ ${ }^{1}$ Department of Dermatology, The Second Affiliated Hospital of Guangzhou Medical University, Guangzhou, China \\ ${ }^{2}$ Department of Dermatology, Zhujiang Hospital, Southern Medical University/The Second School of Clinical Medicine, Guangzhou, China
}

Email address:

mitaiyang@163.com (Xiangbin Mi)

${ }^{*}$ Corresponding author

$\dagger$ Miaoxuan Luo and Shanshan Wei are co-first authors.

\section{To cite this article:}

Miaoxuan Luo, Shanshan Wei, Xiangbin Mi, Tangde Zhang, Wu Zhang. The Regulatory Effect of ERK1 Pathway in the DNA

Hypomethylation of MRL/lpr Mice. International Journal of Immunology. Vol. 9, No. 3, 2021, pp. 41-46. doi: 10.11648/j.iji.20210903.11

Received: July 17, 2021; Accepted: August 9, 2021; Published: August 18, 2021

\begin{abstract}
Background: Defective ERK1 activity may regulate the DNA hypomethylation in T cells of lupus mice. Aims: To investigate the function of ERK1 in regulating DNA hypomethylation and explore the potential molecular mechanisms involved in lupus mice. Methods: $\mathrm{CD}^{+} \mathrm{T}$ cells were isolated from MRL/lpr and $\mathrm{BALB} / \mathrm{c}$ mice, activated in vitro in the presence or absence of 5-azacytidine (5-Aza) (a DNA methyltransferase inhibitor)/U0126 (a selective inhibitor of the ERK signaling pathway) respectively. The positive rate of $\mathrm{CD}^{+} \mathrm{T}$ cell was measured by flow cytometry, and expression levels of CD70, ERK1, DNA transferase 1 (DNMT1) were estimated by RT-PCR and Western blot. The methylated DNA is detected using the Capture and Detection antibodies, then quantified colorimetrically. Results: The expression level of p-ERK1 in MRL/lpr mice was significantly lower than healthy control $(P<0.05)$; Meanwhile, down regulation of DNMT1 and DNA hypomethylation were found in $\mathrm{MRL} / \mathrm{lpr}$ mice $\mathrm{T}$ cells. In contrary, the expression levels of CD70 increased when compared with healthy control $(P<0.05)$. The same changes were seen in healthy $\mathrm{CD}^{+} \mathrm{T}$ cells treated with U0126. Conclusions: DNA hypomethylation regulating mechanisms in lupus $\mathrm{T}$ cells were associated with ERK1 signal pathway. U0126 could inhibit ERK1 signal transduction pathway in normal T cells, and induce DNA hypomethylation by regulating the expression of methylation sensitive gene CD70.
\end{abstract}

Keywords: Systemic Lupus Erythematosus, DNA Methylation, ERK1 Signal Pathway, Epigenetics

\section{Introduction}

DNA methylation of eukaryotic chromosome is a regulatory way of gene expression. In the process of cell growth and differentiation, the role of DNA methylation is to regulate specific gene expression and the mechanism constitutes epigenetic in gene expression. As a way of regulating the gene expression, DNA methylation play an important role in the immune disorder and tumorigenesis. $\mathrm{CpG}$ nucleotides methylation in the promoter region was related to gene transcriptional silence, however, demethylation of the same DNA sequences may induce the transcription of these genes [15]. In vivo, methylation modification of promoters or $\mathrm{CpG}$ base-pairs near the 5' endpoint could inhibit the expression of related genes. SLE is a typical autoimmune disease characterized by abnormal activation of $\mathrm{T}$ and $\mathrm{B}$ lymphocytes and a large number of autoantibodies. A growing number of studies show that hypomethylation of $\mathrm{CD} 4^{+} \mathrm{T}$ lymphocytes play a key role in the development of SLE, and moreover, abnormal epigenetic regulation mechanism in lupus $\mathrm{T}$ lymphocytes may be associated with ERK1 signal pathways. Mitogen-activated protein kinases (MAPKs) signal pathway play a key role in extracellular signals inducted nucleus response, and ERK pathway is an important member of the family of MAPKs $[2,17]$.

In summary, ERK signal pathway defects in $\mathrm{T}$ cell may be involved in the development of autoimmunity and autoantibody production in SLE through reducing DNMT expression and regulating DNA methylation. To the best of our knowledge, there is little data about the ERK signal 
pathway and DNA methylation in T cells of SLE. In the current study, we aimed to investigate the function of ERK1 in regulating DNA hypomethylation and explore the potential molecular mechanisms involved in lupus mice.

\section{Materials and Methods}

\subsection{Mice}

We choosed 10 female 20-weeks MRL/lpr mice (All of them purchased from Nanjing University institute of Animal Models) as Lupus mice model. A total of 30 female 8-weeeks BALB/c mice purchased from Experimental Animal Center of Guangdong Province, were grouped as the healthy control. All $\mathrm{BALB} / \mathrm{c}$ mice were randomly divided into three groups, each group of 10, including U0126 treated group, 5-Aza treated group and the healthy control respectively. This study had approved institutional ethics committee.

\section{2. $\mathrm{CD}^{+} \mathrm{T}$ Cells Separation and Cell Culture}

Peripheral mononuclear blood cell (PBMC) were separated from mice spleen by Red blood cell lysis buffer (Beyotime, China) followed by $\mathrm{CD}^{+} \mathrm{T}$ cell isolation using a MACS magnetic bead $\mathrm{CD}^{+} \mathrm{T}$ cell isolation kit (Miltenyi Biotec, Germany). Purification of $\mathrm{CD}^{+} \mathrm{T}$ cells were measured by flow cytometry. All $\mathrm{CD}^{+} \mathrm{T}$ cells were stimulated with 1 $\mu \mathrm{g} / \mathrm{mL}$ phytohemagglutinin (PHA) (Sigma, USA) in 10\% fetal calf serum RPMI-1640 for $24 \mathrm{~h}$ and then expanded with 50
U/mL IL-2 (PeproTech, USA) for an additional 72 h. CD4 ${ }^{+} \mathrm{T}$ cells from the healthy BALB/c mice were then divided into three groups (U0126 group, 5-Aza group and control group). Following activation with PHA, the U0126 treated group were expanded with IL-2 and $5 \mu \mathrm{mol} / \mathrm{mL} \mathrm{U0126}$ (Beyotime, China), the 5-Aza treated group were expanded with IL-2 and 1 $\mu \mathrm{mol} / \mathrm{mL} 5$-Aza, while the healthy control was expanded with IL-2 alone.

\subsection{Cell Viability}

We used cell counting kit-8 assay (CCK8) (Dojindo Molecular Technologies, Tokyo, Japan) to measure cell viability. Cells were prepared in 96-well cell culture plates at a density of $5 \times 10^{4}$ cells/well. The cell suspension was centrifuged to collect cells, then cells were washed three times with PBS, then a 1:10 diluted CCK8 solution in RPMI 1640 was added to the cells and incubated for $2 \mathrm{~h}$ at $37^{\circ} \mathrm{C}$. The results were measured by a microplate reader at $450 \mathrm{~nm}$.

\subsection{Real-time Quantitative Polymerase Chain Reaction (qPCR)}

qPCR was used to quantify the mRNA expression level of ERK1, DNMT1 and CD70. qPCR analyses were conducted in triplicate for each sample. The specific primers used for amplification were showed in Table 1. All primers were synthetized by Sango Biotech (China, Shanghai).

Table 1. Primers used for real-time PCR.

\begin{tabular}{lll}
\hline \multirow{2}{*}{ Name } & Primers & \\
\cline { 2 - 3 } & Forward & Reverse \\
\hline ERK1 & 5'-TCCGCCATGAGAATGTTATAGGC-3' & 5'-GGTGGTGTTGATAAGCAGATTGG-3' \\
DNMT1 & 5'-AAGAATGGTGTTGTCTACCGAC-3' & 5'-CATCCAGGTTGCTCCCCTTG-3' \\
CD70 & 5'-AGGAGGGCCATCTGCGTAT-3' & 5'-AGGCGCTGTAATGCCACTG-3' \\
GADPH & 5'-AGGTCGGTGTGAACGGATTTG-3' & 5'-GGGGTCGTTGATGGCAACA-3' \\
\hline
\end{tabular}

\subsection{DNA Extraction and Methylated DNA Quantification}

The genomic DNA was precipitate from the interphase and organic phase as instructed by the manufacturer. An aliquot of the DNA preparation was measured with micro drop and the DNA concentration was calculated using the A260 value for double-stranded DNA. The level of methylated DNA is quantified colorimetrically with an imprint methylated DNA quantification kit (Sigma, Spruce Street, St Louis, USA) as instructed by the manufacturer.

\subsection{Western Blot}

$\mathrm{CD} 4{ }^{+} \mathrm{T}$ cells were lysed in RIPA lysis buffer (cwbiotech, China). Protein concentration was determined by BCA assay (pierce, USA). The following primary antibodies were used: Rabbit monoclonal antibody to GAPDH (Pierce, America), rabbit monoclonal antibody to ERK1 (Cell signaling, America), rabbit monoclonal antibody to p-ERK1 (Epitomics, America), rabbit monoclonal antibody to DNMT1 (Cell Signaling, America), Rabbit polyclonal antibody to CD70 (Abcam,
America). Bands were examined by scan densitometry using an intelligent imaging system (Syngene, UK).

\subsection{Statistical Analysis}

Student's $t$-test and $\chi 2$ were used to analyze the differences between groups. Data are denoted in terms of the mean \pm SD. All statistical analyses were performed using SPSS 22.0 software (IBM Corp., USA). The Graphs were produced by GraphPad Prism 5.0 Software. Here as well, $P<0.05$ was considered statistically significant.

\section{Results}

\subsection{ERK1 Signaling Pathway Defects in MRL/pr Mice CD4 ${ }^{+}$T Cells}

ERK pathway is an important member of the family of MAPKs which play a key role in extracellular signals, and the expression level of phosphorylated ERK1 and ERK1 were abnormal in many autoimmune diseases. Therefore, we investigated whether there was an abnormal expression in 
lupus mice. As shown in Figures 1 and 2, the MRL/lpr group has a higher $\mathrm{p}$-ERK/ERK ratio compared with the control group, suggesting the abnormal activation of ERK1 in lupus mice. Then $\mathrm{CD}^{+} \mathrm{T}$ cells were isolated from BALB/c mice, and activated in the presence of 5-Aza and /U0126 respectively, the results showed that treatment with U0126 or 5 -Aza resulted in significantly lower expression of p-ERK1/ERK1 compared with the control group $(P<0.05)$, however, there was no significant differences when compared with lupus $\mathrm{CD}^{+} \mathrm{T}$ cells $(P>0.05)$.

\subsection{Lower Expression of DNMT1 and DNA Hypomethylation Were Showed in $\mathrm{CD}^{+} \mathrm{T}$ Cells of MRL/lpr Mice}

Previous studies found DNMT was regulated by ras-mitosis activated protein kinase signaling pathway, and the inhibition of c-jun NH2 could reduce the expression of DNMT1 and DNMT3a [19]. In this study, the MRL/lpr group had a lower expression of DNMT1 and DNA hypomethylation compared with the control group, and treatment with 5-Aza/U0126 resulted in significantly lower expression of DNMT1 and genome-wide DNA hypomethylation as compared to the control group (Figure 3, $P<0.05$ ). The same changes were seen in MRL/lpr, 5-Aza and U0126 group $(P>0.05)$.

\subsection{CD4 ${ }^{+}$T Cells of MRL/lpr Mice over Express CD70, a Methylation Sensitive Gene}

In recent years, researchers found that abnormalities of multiple cytokines and cell activation related surface molecular played an important role in SLE development and prognosis, and excessive expression of CD70, CD11a and perforin contributed to $\mathrm{T}$ cells auto-reaction, $\mathrm{B}$ cell higher activation and abnormality of phagocytes cracking dysfunction respectively [19]. The excessive expression of these genes was closely related to cytosine hypomethylation of the gene regulation area. Then, we detected the expression of CD70 in $\mathrm{CD}^{+} \mathrm{T}$ cells in different groups respectively. The U0126 and 5-Aza group had an overexpress of CD70 compared with the control group $(P<0.05)$. The same changes were also seen in MRL/lpr mice, and differences among the MRL/lpr, U0126 and 5-Aza group had no statistical significance $(P>0.05)$.

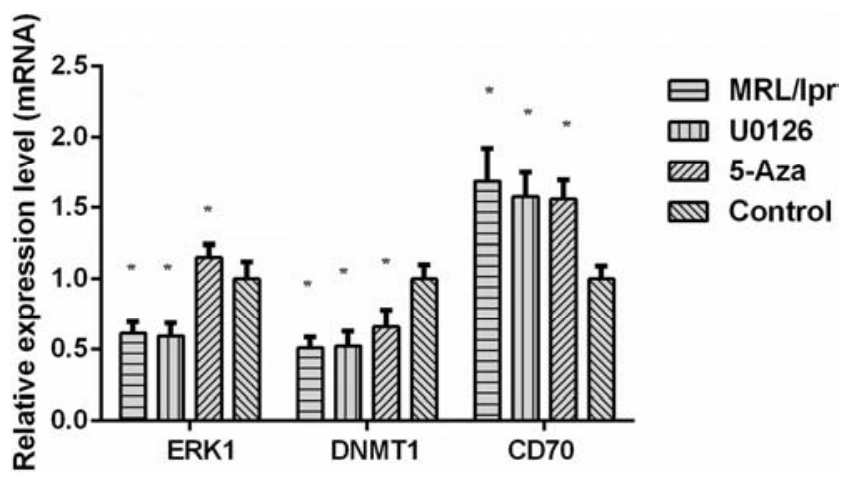

Figure 1. The CD70, DNMT1, ERK1 mRNA in different groups. ${ }^{*} P<0.05$, compared with the untreated group.
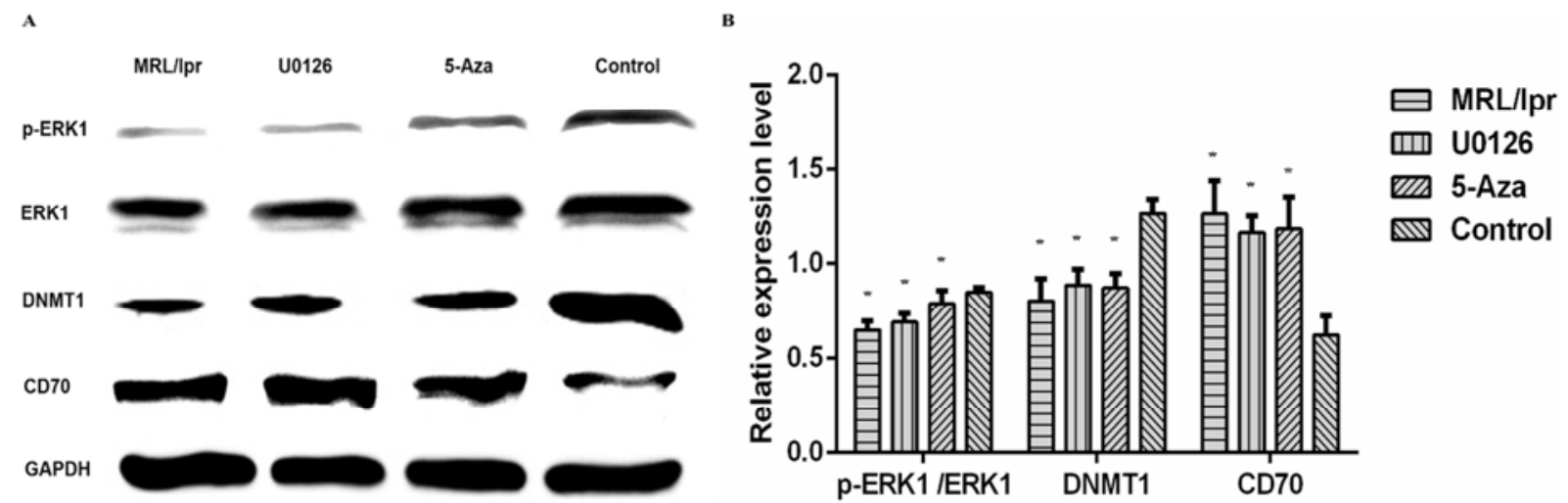

Figure 2. The level of CD70, DNMT1, pERK1, ERK1 protein in different groups. (A) Cells were incubated in the absence or presence of U0126, 5-Aza and analyzed for the proteins expression levels as indicated. (B) Densitometric analysis of the CD70, DNMT1, $p$-ERK1/ERK1 ratio from different group. ${ }^{*} P<0.05$, compared with the control group.

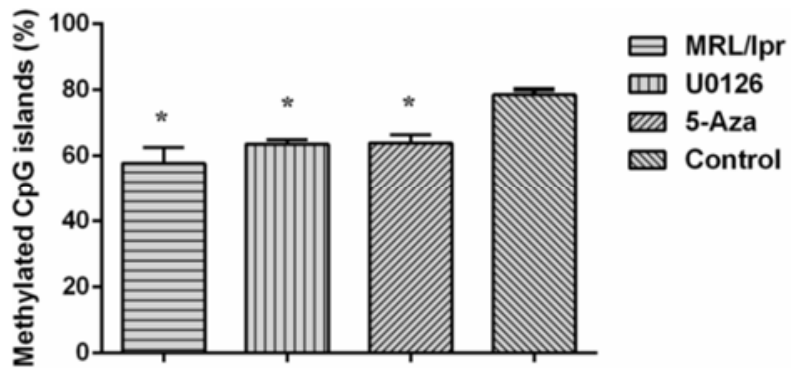

Figure 3. Global DNA methylation levels of peripheral blood $C D 4^{+}$T cells in the MRL/lpr, U0126, 5-Aza group significantly decreased when compared with the control group. ${ }^{*} P<0.05$, compared with the control group.

\subsection{Relationship of ERK1, DNMT1 and CD70}

In this experiment, we analyzed the correlation of gene and protein expression level of ERK1, DNMT1 and CD70 in lupus $\mathrm{T}$ cells. Results showed that CD70 expression levels were negative correlated to ERK1 and DNMT1 in peripheral blood CD4 + T cells of MRL/lpr mice; on the contrary, the expression levels of ERK1 and DNMT1 showed a positive correlation relationship (Figure 4).

Similarly, CD70 expression levels were negative correlated to ERK1 and DNMT1 in U0126 treated CD4 ${ }^{+}$T cells of BALB/c mice; on the contrary, the expression levels of ERK1 and 
DNMT1 showed a positive correlation relationship. These results indicated that ERK signal pathway defects might suppress
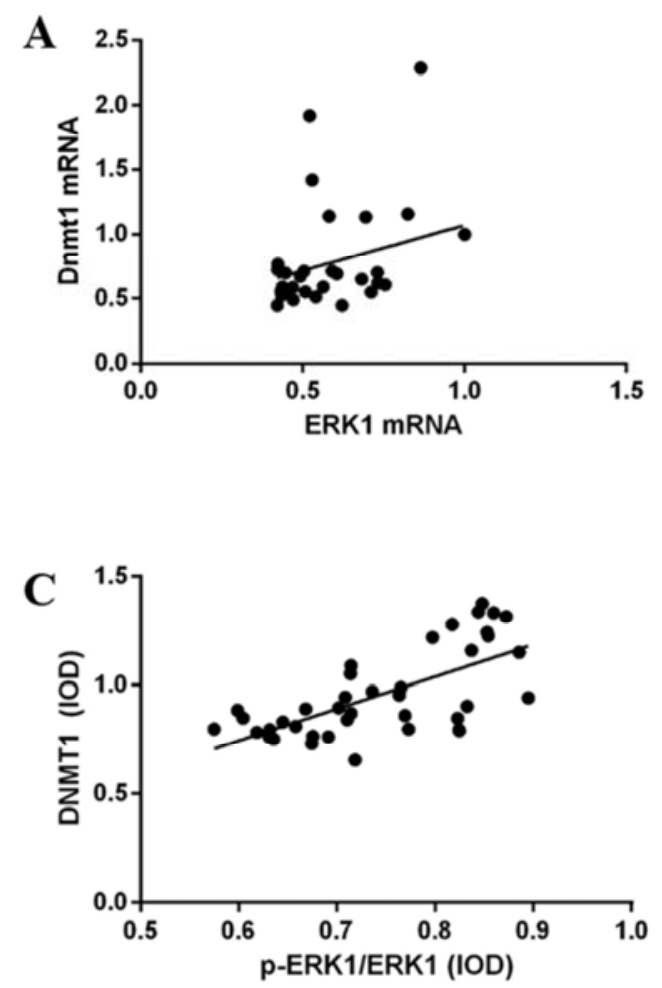

the Dnmt1 gene expression and reduce the DNA methylation level, and then regulate methylation sensitive gene expression.
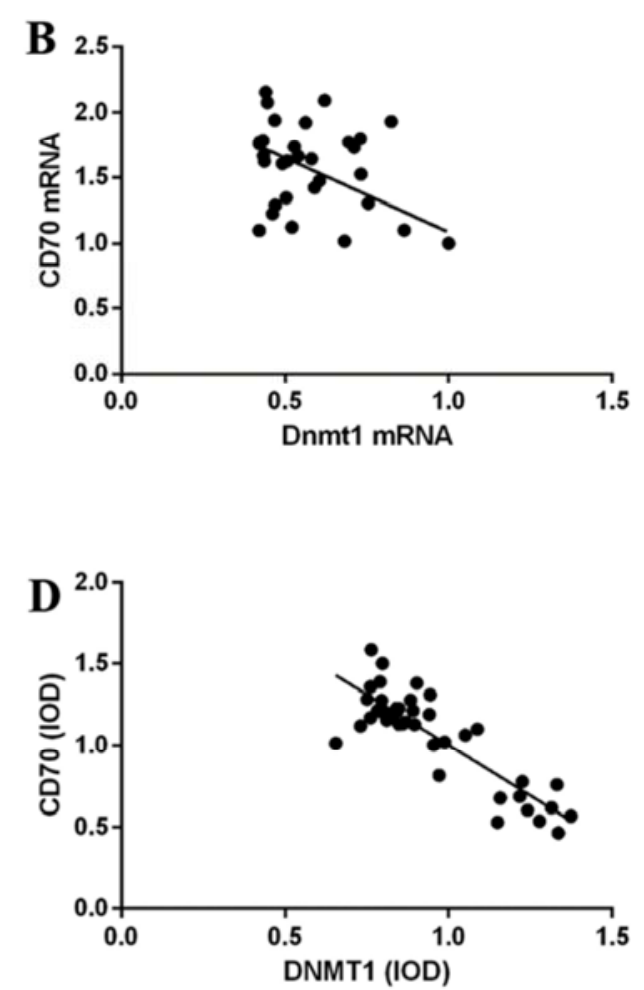

Figure 4. The correlation of ERK1, DNMT1 and CD70 in CD4 $4^{+}$cells.

\section{Discussion}

Systemic lupus erythematosus was more common in childbearing age women, and the morbidity of female and male was about 9:1. SLE could successively or simultaneously affect the multiple systems and life quality of the patient. With the development of science and experimental technology, studies on this disease from clinic gradually went deep into the level of molecular biology and were expected to further clarify the pathogenesis from the epigenetics defects in the pathogenesis of SLE [14]. Epigenetics is a kind of heritable changes in gene expression, and the DNA sequence does not change, including DNA methylation which mainly located in the transcriptional promoter $\mathrm{CpG}$ dinucleotide sites, histone modification and abnormal expression of miRNAs [4] $\mathrm{CpG}$ methylation leads to transcription inhibition of its downstream genes, while $\mathrm{CpG}$ demethylation leads to excessive expression of its downstream genes which mediated by DNMT1, DNMT3A and DNMT3B [16]. DNMT3A and DNMT3B participated in de novo synthesis of DNA methylation, and meanwhile DNMT1 mainly synthesized methylated DNA using half-methylated DNA as substrate which worked together to maintain the status of DNA methylation $[6,8]$.

At first, DNA methylation was considered to be a simple genetic modification method which inhibited gene transcription through methyl group connection. However, more and more data suggested DNA methylation played an important role in many diseases such as autoimmune diseases and tumor [12]. Multiple immune abnormalities existed in lupus $\mathrm{T}$ cells, and they formed the basis of SLE pathology which DNA methylation was the most characteristic [3].

Results of this experiment showed that genome-wide methylation level and DNMT1 expression level decreased obviously in MRL/lpr mice peripheral blood $\mathrm{CD} 4^{+} \mathrm{T}$ cells; the same changes were seen in 5-Aza treated group which indicating DNA methylation may be involved in the SLE pathogenesis. CD70, which was known as one of the methylation sensitive genes, was ligand of CD27, a member of tumor necrosis factor (TNF) family. Studies found that excessive expression of CD70 could enhance B cell activity and promote its production of autoantibodies which positively related to SLE disease activity [19]. Our results showed that CD70 expression increased in 5-Aza treated CD4 ${ }^{+} \mathrm{T}$ cells and $\mathrm{MRL} / \mathrm{lpr}$ mice, moreover, it was negatively correlated to expression level of DNMT1.

In recent years, some scholars proposed epigenetic defects in lupus T cells may be related to many signaling transduction [10]. Mitogen-activated protein kinases (MAPKs) signal pathway may play a key role in the pathogenesis of SLE [2]. Four MAPK cascades have been defined based on the components in the MAPK layer: ERK1/2, c-Jun N-terminal kinase (JNK), p38 MAPK and ERK5, and ERK1/2 regulated the cell divisional activation and survival rate of cells [13]. Research showed that phosphorylated ERK1/2 decreased in peripheral blood $\mathrm{T}$ cells of active SLE patients when compared with the healthy control group, and the expression 
level of active ERK1/2 negatively correlated with disease activity [11]. Our experiment results show that the expression level- of $\mathrm{p}$-ERK1 declined in $\mathrm{CD} 4^{+} \mathrm{T}$ cells from MRL/lpr mice and U0126 treated group. The expression level of p-ERK1 was positively related with DNMT1 and negatively related with CD70, a methylation-sensitive gene.

The expression of DNMT could be regulated by ras-mitosis activated protein kinase signaling pathway. Inhibition of c-jun $\mathrm{NH}_{2}$ terminal kinase or extracellular signal regulating kinase pathway could reduce the expression of DNMT1 and DNMT3a. The c-jun $\mathrm{NH}_{2}$ terminal kinase signaling pathways in lupus $\mathrm{T}$ cell is normal, while ERK signal decreased which directly related to disease activity. Study have found that the MEK/ERK signal pathway may affect the level of DNA methylation through regulating the expression of DNMT1 [5, 9]. In addition, mesenchymal stem cells activate the MEK/ERK signaling pathway and enhance DNA Methylation via DNMT1 in PBMC from Systemic Lupus Erythematosus [18]. Combined with our experiment results, we concluded that ERK signaling pathway defects could inhibit DNMT1 expression, decrease DNA methylation level and regulate methylation sensitive gene expression which leads to cytokine disorder and thus promote the development of SLE. Bloch O etal found that the expression level of ERK decreased and p-ERK increased in SLE patients. The cause of results was continuing abnormal activation of ERK led to depletion of ERK [1]. In our study, we found the expression level of p-ERK/ERK was decreased in SLE patients. Here are several possible reasons: first, SLE is a kind of autoimmune disease which involve multiple systems and organs which affected by heredity, sex hormone levels, environment, drugs and even virus infection, and there may be existing a great individual difference; second, SLE patients had a long duration, so at various stages of the disease the pathological reaction may be different.

\section{Conclusion}

There was certain inner link between ERK signal pathway and DNA epigenetic characteristics in MRL/lpr mice. DNA hypomethylation regulating mechanisms in lupus $\mathrm{T}$ cells were associated with ERK1 signal pathway. U0126 could inhibit ERK1 signal transduction pathway in normal $\mathrm{T}$ cells, and induce DNA hypomethylation by regulating the expression of methylation sensitive gene CD70. ERK signal transduction pathway may play a key role in SLE epigenetic abnormality through DNA hypomethylation.

\section{Funding}

The present study was supported by the National Science Foundation of China (Grant No. 81201233 and Grant No. 81803119).

\section{Competing Interests}

The authors declare that they have no competing interests.

\section{Availability of Data and Materials}

The datasets generated and/or analyzed during the present study are available from the corresponding author on reasonable request.

\section{Authors' Contributions}

XBM conceived and designed the study. MXL, SSW and XBM performed the experiments. MXL and TDZ analyzed the data. MXL, SSW and WZ wrote the manuscript. All authors read and approved the final manuscript.

\section{Ethics Approval and Consent to Participate}

The protocols of the present study were approved by the Ethics Committee of Southern Medical University.

\section{References}

[1] Bloch, O., Amit-Vazina, M., Yona, E., Molad, Y., \& Rapoport, M. J. (2014). Increased ERK and JNK activation and decreased ERK/JNK ratio are associated with long-term organ damage in patients with systemic lupus erythematosus [Journal Article; Multicenter Study]. Rheumatology (Oxford), 53 (6), 1034-1042. http://doi.org/10.1093/rheumatology/ket482

[2] Chuang, H. C., \& Tan, T. H. (2019). MAP4K Family Kinases and DUSP Family Phosphatases in T-Cell Signaling and Systemic Lupus Erythematosus [Journal Article; Review; Research Support, Non-U.S. Gov't]. Cells, 8 (11) http://doi.org/10.3390/cells8111433

[3] Hurtado, C., Acevedo, S. L., Vasquez, T. E., Urrego, R., Jenks, S., Sanz, I., \& Vasquez, G. (2020). DNA methylation changes on immune cells in Systemic Lupus Erythematosus [Journal Article]. AUTOIMMUNITY, 1-8. http://doi.org/10.1080/08916934.2020.1722108

[4] Imgenberg-Kreuz, J., Carlsson, A. J., Leonard, D., Alexsson, A., Nordmark, G., Eloranta, M. L., Rantapaa-Dahlqvist, S., Bengtsson, A. A., Jonsen, A., Padyukov, L., Gunnarsson, I., Svenungsson, E., Sjowall, C., Ronnblom, L., Syvanen, A. C., \& Sandling, J. K. (2018). DNA methylation mapping identifies gene regulatory effects in patients with systemic lupus erythematosus [Journal Article; Research Support, Non-U.S. Gov't]. ANNALS OF THE RHEUMATIC DISEASES 77 (5), 736-743. http://doi.org/10.1136/annrheumdis-2017-212379

[5] Li, Y., Gorelik, G., Strickland, F. M., \& Richardson, B. C. (2014). Oxidative stress, T cell DNA methylation, and lupus [Journal Article; Research Support, N. I. H., Extramural; Research Support, U.S. Gov't, Non-P.H.S.]. Arthritis \& Rheumatology, $66 \quad$ (6), 1574-1582. http://doi.org/10.1002/art.38427

[6] Liang, J., Zhu, X. H., Qin, H. H., Lin, J. R., Wang, D. Q., Huang, L., Luo, X. Q., \& Xu, J. H. (2015). A correlation study on the effects of DNMT1 on methylation levels in CD4(+) T cells of SLE patients [Journal Article]. International Journal of Clinical and Experimental Medicine, 8 (10), 19701-19708. 
[7] Liao, W., Li, M., Wu, H., Jia, S., Zhang, N., Dai, Y., Zhao, M., \& Lu, Q. (2017). Down-regulation of MBD4 contributes to hypomethylation and overexpression of CD70 in CD4(+) T cells in systemic lupus erythematosus [Journal Article; Research Support, Non-U.S. Gov't]. Clinical Epigenetics, 9, 104. http://doi.org/10.1186/s13148-017-0405-8

[8] Nawrocki, M. J., Majewski, D., Puszczewicz, M., \& Jagodzinski, P. P. (2017). Decreased mRNA expression levels of DNA methyltransferases type 1 and $3 \mathrm{~A}$ in systemic lupus erythematosus [Journal Article]. RHEUMATOLOGY INTERNATIONAL, $\quad 37 \quad$ (5), 775-783. http://doi.org/10.1007/s00296-017-3711-8

[9] Pradhan, N., Parbin, S., Kar, S., Das, L., Kirtana, R., Suma, S. G., Sengupta, D., Deb, M., Kausar, C., \& Patra, S. K. (2019). Epigenetic silencing of genes enhanced by collective role of reactive oxygen species and MAPK signaling downstream ERK/Snail axis: Ectopic application of hydrogen peroxide repress $\mathrm{CDH} 1$ gene by enhanced DNA methyltransferase activity in human breast cancer [Journal Article; Research Support, Non-U.S. Gov't]. Biochim Biophys Acta Mol Basis Dis, 1865 (6), 1651-1665. http://doi.org/10.1016/j.bbadis.2019.04.002

[10] Qiu, H., Wu, H., Chan, V., Lau, C. S., \& Lu, Q. (2017). Transcriptional and epigenetic regulation of follicular T-helper cells and their role in autoimmunity [Journal Article; Review]. AUTOIMMUNITY, $\quad 50 \quad$ 71-81. http://doi.org/10.1080/08916934.2017.1284821

[11] Sakurai, D., Zhao, J., Deng, Y., Kelly, J. A., Brown, E. E., Harley, J. B., Bae, S. C., Alarcomicronn-Riquelme, M. E., Edberg, J. C., Kimberly, R. P., Ramsey-Goldman, R., Petri, M. A., Reveille, J. D., Vila, L. M., Alarcon, G. S., Kaufman, K. M., Vyse, T. J., Jacob, C. O., Gaffney, P. M., Sivils, K. M., James, J. A., Kamen, D. L., Gilkeson, G. S., Niewold, T. B., Merrill, J. T., Scofield, R. H., Criswell, L. A., Stevens, A. M., Boackle, S. A., Kim, J. H., Choi, J., Pons-Estel, B. A., Freedman, B. I., Anaya, J. M., Martin, J., Yu, C. Y., Chang, D. M., Song, Y. W., Langefeld, C. D., Chen, W., Grossman, J. M., Cantor, R. M., Hahn, B. H., \& Tsao, B. P. (2013). Preferential binding to Elk-1 by SLE-associated IL10 risk allele upregulates IL10 expression [Journal Article; Research Support, N. I. H., Extramural; Research Support, Non-U.S. Gov't; Research Support, U.S. Gov't, Non-P.H.S.]. PLoS $\begin{array}{llll}\text { Genetics, } & 9 & (10), & \text { el003870. }\end{array}$ http://doi.org/10.1371/journal.pgen.1003870
[12] Salameh, Y., Bejaoui, Y., \& El, H. N. (2020). DNA Methylation Biomarkers in Aging and Age-Related Diseases [Journal Article; Review]. Frontiers in Genetics, 11, 171. http://doi.org/10.3389/fgene.2020.00171

[13] Tanimura, S., \& Takeda, K. (2017). ERK signalling as a regulator of cell motility [Journal Article; Review]. JOURNAL $\begin{array}{llll}\text { OF } & \text { BIOCHEMISTRY, } 162 & \text { (3), } & 145-154 .\end{array}$ http://doi.org/10.1093/jb/mvx048

[14] Tsokos, G. C., Lo, M. S., Costa, R. P., \& Sullivan, K. E. (2016). New insights into the immunopathogenesis of systemic lupus erythematosus [Journal Article; Review]. Nature Reviews Rheumatology, 12 (12), 716-730. http://doi.org/10.1038/nrrheum.2016.186

[15] Tsukakoshi, K., Saito, S., Yoshida, W., Goto, S., \& Ikebukuro, K. (2018). CpG Methylation Changes G-Quadruplex Structures Derived from Gene Promoters and Interaction with VEGF and SP1 [Journal Article]. MOLECULES, 23 (4) http://doi.org/10.3390/molecules23040944

[16] Wardowska, A. (2020). The epigenetic face of lupus: Focus on antigen-presenting cells [Journal Article; Review]. INTERNATIONAL IMMUNOPHARMACOLOGY, 81, 106262. http://doi.org/10.1016/j.intimp.2020.106262

[17] Wu, W., Jing, Y., Xu, Q., Hao, J., \& Yu, X. (2020). Upregulated level of lncRNA HOXA-AS2 in peripheral blood of systemic lupus erythematosus patients aggravates disease progression via ERK pathway [Journal Article]. MINERVA MEDICA http://doi.org/10.23736/S0026-4806.19.06361-4

[18] Xiong, H., Guo, Z., Tang, Z., Ai, X., Qi, Q., Liu, X., Huang, D., Li, Z., Ji, S., \& Guo, Q. (2020). Mesenchymal Stem Cells Activate the MEK/ERK Signaling Pathway and Enhance DNA Methylation via DNMT1 in PBMC from Systemic Lupus Erythematosus [Journal Article]. Biomed Research International, $2020, \quad 4174082$. http://doi.org/10.1155/2020/4174082

[19] Zhang, C., Wang, X., Chen, Y., Wu, Z., Zhang, C., \& Shi, W. (2018). The down-regulation of hsa circ 0012919, the sponge for miR-125a-3p, contributes to DNA methylation of CD11a and CD70 in CD4(+) T cells of systemic lupus erythematous [Journal Article; Research Support, Non-U.S. Gov't]. Clin Sci (Lond), $\quad 132 \quad$ (21), 2285-2298. http://doi.org/10.1042/CS20180403 